PROCEEDINGS OF THE

AMERICAN MATHEMATICAL SOCIETY

Volume 126, Number 3, March 1998, Pages 827-834

S $0002-9939(98) 04224-5$

\title{
RADIATION CONDITIONS AND UNIQUENESS FOR STATIONARY OSCILLATIONS IN ELASTIC PLATES
}

\author{
CHRISTIAN CONSTANDA
}

(Communicated by Palle E. T. Jorgensen)

\begin{abstract}
Sommerfeld-type radiation conditions are indicated for the solutions of the system governing the small stationary oscillations in plates with transverse shear deformation, and a uniqueness theorem is proved in the case of the corresponding exterior Dirichlet and Neumann problems.
\end{abstract}

\section{Preliminaries}

In what follows Greek and Latin suffixes are assumed to take the values 1,2 and $1,2,3$, respectively, and the superscript $\mathrm{T}$ denotes matrix transposition. If $L$ is a scalar operator and $\Psi$ a vector function, then $L \Psi$ means that $L$ is applied to each component of $\Psi$. Also, if $X$ is a space of scalar functions, $\Psi \in X$ means that each component of $\Psi$ belongs to $X$.

Let $\Omega$ be a domain in $\mathbb{R}^{3}$ bounded by a closed surface $\partial \Omega$, which is occupied by an isotropic and homogeneous elastic material of Lamé coefficients $\lambda$ and $\mu$ and of density $\rho$. Also, let $x=\left(x_{1}, x_{2}, x_{3}\right)^{\mathrm{T}}$ be the position vector of a generic point in $\Omega$. The equations governing the small dynamic deformations of this body can be written in the form $[1$, Ch. I, $\S 11.1]$

$$
\mu \Delta \Phi+(\lambda+\mu) \operatorname{grad} \operatorname{div} \Phi+\rho F=\rho \ddot{\Phi},
$$

where $\Phi=\left(\Phi_{1}, \Phi_{2}, \Phi_{3}\right)^{\mathrm{T}}$ is the displacement vector and two superposed dots denote the second order time-derivative. If the body force $F$ is of the form

$$
F(x, t)=\operatorname{Re}\left[f(x) e^{-i \omega t}\right],
$$

where $f$ is a complex-valued vector function, and if the boundary conditions (prescribed on $\partial \Omega$ ) have similar expressions, then the body performs stationary oscillations of frequency $\omega$ and its expected displacements are of the form

$$
\Phi(x, t)=\operatorname{Re}\left[\varphi(x) e^{-i \omega t}\right],
$$

with $\varphi=\left(\varphi_{1}, \varphi_{2}, \varphi_{3}\right)^{\mathrm{T}}$ also complex-valued and satisfying

$$
\mu \Delta \varphi+(\lambda+\mu) \operatorname{grad} \operatorname{div} \varphi+\rho \omega^{2} \varphi+\rho f=0 .
$$

In the case of an elastic plate we have $\Omega=S \times\left(-h_{0} / 2, h_{0} / 2\right)$, where $S$ is a domain in $\mathbb{R}^{2}$ bounded by a closed contour $\partial S$ and $h_{0}=$ const $\ll \operatorname{diam} S$. Owing to this

Received by the editors April 30, 1996 and, in revised form, September 9, 1996.

1991 Mathematics Subject Classification. Primary 35J55, 73K10, 73C15, 73D30.

This work was supported in part by a grant from the Carnegie Trust for the Universities of Scotland.

(C)1998 American Mathematical Society 
special geometry, the study of bending of plates makes use of certain simplifying assumptions. Thus, when transverse shear deformation is taken into account, the fundamental (kinematic) assumption is that the displacement field is of the form

$$
\varphi_{\beta}=x_{3} u_{\beta}\left(x_{1}, x_{2}\right), \quad \varphi_{3}=u_{3}\left(x_{1}, x_{2}\right) .
$$

Let $x=\left(x_{1}, x_{2}\right)^{\mathrm{T}}$ be the position vector of a generic point in $S$, and let $w=$ $\left(u_{1}, u_{2}, 0\right)^{\mathrm{T}}$. If the body forces and moments, and the forces and moments acting on the faces $x_{3}= \pm h_{0} / 2$, are negligible, then the usual procedure of averaging (1) over the thickness of the plate $[2, \S 2.1]$ leads to the system of equations

$$
\begin{gathered}
h^{2}(\lambda+\mu) \operatorname{grad} \operatorname{div} w+h^{2} \mu \Delta w+\mu \theta w-\mu \operatorname{grad} u_{3}=0, \\
\left(\Delta+k^{2}\right) u_{3}+\operatorname{div} w=0,
\end{gathered}
$$

where $h^{2}=h_{0}^{2} / 12, k^{2}=\rho \omega^{2} / \mu$ and $\theta=k^{2} h^{2}-1$.

A solution $u=w+\left(0,0, u_{3}\right)^{\mathrm{T}}$ of $(2)$ is called regular if $u \in C^{2}(S) \cap C^{1}(\bar{S})$. From now on we assume that

$$
\lambda+\mu>0, \quad \mu>0, \quad \omega>\frac{1}{h} \sqrt{\frac{\mu}{\rho}} .
$$

The first two conditions ensure that (2) is elliptic; the last one is equivalent to $k^{2} h^{2}>1$.

We denote by $S^{+}$the bounded domain enclosed by $\partial S$, write $S^{-}=\mathbb{R}^{2} \backslash\left(S^{+} \cup \partial S\right)$, and assume that $\partial S$ has a continuous normal of unit outward (with respect to $S^{+}$) vector $\nu=\left(\nu_{1}, \nu_{2}\right)^{\mathrm{T}}$.

The exterior Dirichlet and Neumann problems $\left(\mathrm{D}^{-}\right)$and $\left(\mathrm{N}^{-}\right)$consist in finding a solution of (2) in $S^{-}$which takes prescribed values on $\partial S$, or for which the bending and shear stress vector $T u[3]$ takes prescribed values on $\partial S$, respectively.

In [3] radiation conditions were indicated under which $\left(\mathrm{D}^{-}\right)$and $\left(\mathrm{N}^{-}\right)$have at most one regular solution. Unfortunately, these conditions are not of the form one might expect for stationary oscillations. In what follows we intend to sharpen considerably the results of the analysis in [3] and give full Sommerfeld-type conditions that imply uniqueness for the solutions of these boundary value problems.

System (2) describes the small stationary flexural oscillations of a plate, and its solutions play an important role in the study of scattering of elastic waves that occur, for example, in the process of non-destructive testing of plate-like structures.

\section{RADIATION CONDITIONS}

Setting

$$
\begin{gathered}
\mu \theta w^{(1)}=-h^{2}(\lambda+2 \mu) \operatorname{grad} \operatorname{div} w+\mu \operatorname{grad} u_{3}, \\
\theta w^{(2)}=h^{2} \operatorname{curl} \operatorname{curl} w
\end{gathered}
$$

we arrive at the representation [3]

$$
w=w^{(1)}+w^{(2)},
$$

where

$$
\begin{gathered}
\left(\Delta+k_{1}^{2}\right)\left(\Delta+k_{2}^{2}\right) w^{(1)}=0, \\
\left(\Delta+k_{3}^{2}\right) w^{(2)}=0
\end{gathered}
$$


and

$$
\begin{gathered}
\operatorname{curl} w^{(1)}=0, \\
\operatorname{div} w^{(2)}=0 .
\end{gathered}
$$

Also, $u_{3}$ satisfies the equation

$$
\left(\Delta+k_{1}^{2}\right)\left(\Delta+k_{2}^{2}\right) u_{3}=0 .
$$

Here $k_{1}^{2}, k_{2}^{2}$ and $k_{3}^{2}$ are defined by

$$
\begin{gathered}
k_{1}^{2}+k_{2}^{2}=\frac{k^{2}(\lambda+3 \mu)}{\lambda+2 \mu}, \quad k_{1}^{2} k_{2}^{2}=\frac{\mu k^{2}\left(k^{2} h^{2}-1\right)}{h^{2}(\lambda+2 \mu)}, \\
k_{3}^{2}=k^{2}-\frac{1}{h^{2}} .
\end{gathered}
$$

In view of (3), all of $k_{1}^{2}, k_{2}^{2}$ and $k_{3}^{2}$ are strictly positive and distinct.

From $(2)_{2}$ and $(6)_{2}$ it follows that

$$
\left(\Delta+k^{2}\right) u_{3}+\operatorname{div} w^{(1)}=0 .
$$

The fact that $w^{(1)}$ and $u_{3}$ do not satisfy Helmholtz equations is highly unsatisfactory. However, this drawback is easily eliminated.

Theorem 1. Any regular solution of (2) can be written in the form

$$
u=U^{(1)}+U^{(2)}+U^{(3)}+v^{(1)}+v^{(2)},
$$

where $U_{3}^{(j)}=0, v_{\gamma}^{(\beta)}=0$ and

$$
\begin{gathered}
\left(\Delta+k_{j}^{2}\right) U^{(j)}=0, \quad \operatorname{curl} U^{(\beta)}=0, \quad \operatorname{div} U^{(3)}=0, \\
\left(\Delta+k_{\beta}^{2}\right) v^{(\beta)}=0 .
\end{gathered}
$$

Proof. Setting

$$
U^{(1)}=-\frac{\left(\Delta+k_{2}^{2}\right) w^{(1)}}{k_{1}^{2}-k_{2}^{2}}, \quad U^{(2)}=\frac{\left(\Delta+k_{1}^{2}\right) w^{(1)}}{k_{1}^{2}-k_{2}^{2}}, \quad U^{(3)}=w^{(2)}
$$

and

$$
v^{(\beta)}=\left(0,0, u_{3}^{(\beta)}\right)^{\mathrm{T}}
$$

where

$$
u_{3}^{(1)}=-\frac{\left(\Delta+k_{2}^{2}\right) u_{3}}{k_{1}^{2}-k_{2}^{2}}, \quad u_{3}^{(2)}=\frac{\left(\Delta+k_{1}^{2}\right) u_{3}}{k_{1}^{2}-k_{2}^{2}}
$$

we easily see that

$$
w^{(1)}=U^{(1)}+U^{(2)}, \quad u_{3}=u_{3}^{(1)}+u_{3}^{(2)}
$$

and that, by (5)-(7) and (4), equalities (11) and (12) are satisfied.

Consider a circular disk $K_{R}$ with the centre at the origin (chosen in $S^{+}$) and radius $R$ sufficiently large so that $\partial S$ lies strictly inside the circumference $\partial K_{R}$. 
In view of the radiation conditions for the Helmholtz equation in the plane [4, 228$]$, equations $(11)_{1}$ and (12) now suggest that, as $R \rightarrow \infty$, the radiation conditions that are appropriate for a regular solution of (2) in $S^{-}$are

$$
\begin{aligned}
& U^{(j)}=O\left(R^{-1 / 2}\right), \quad \frac{\partial U^{(j)}}{\partial R}-i k_{j} U^{(j)}=O\left(R^{-3 / 2}\right), \\
& u_{3}^{(\beta)}=O\left(R^{-1 / 2}\right), \quad \frac{\partial u_{3}^{(\beta)}}{\partial R}-i k_{\beta} u_{3}^{(\beta)}=O\left(R^{-3 / 2}\right) .
\end{aligned}
$$

\section{Asymptotic estimates}

In what follows we assume that $x \in \partial K_{R}, x=\left(x_{1}, x_{2}, 0\right)^{\mathrm{T}}$, and write $R_{0}=x / R$.

Theorem 2. For $R$ large, a regular solution $u$ of $(2)$ in $S^{-}$which satisfies the radiation conditions (14) and (15) also satisfies the estimates

$$
\begin{gathered}
R_{0} \times U^{(\beta)}=O\left(R^{-3 / 2}\right), \\
\operatorname{div} U^{(\beta)}-i k_{\beta} R_{0} \cdot U^{(\beta)}=O\left(R^{-3 / 2}\right), \\
R_{0} \cdot U^{(3)}=O\left(R^{-3 / 2}\right), \\
\operatorname{curl} U^{(3)}-i k_{3} R_{0} \times U^{(3)}=O\left(R^{-3 / 2}\right), \\
U^{(\beta)}=i k_{\beta}^{-1}\left(k^{2}-k_{\beta}^{2}\right) u_{3}^{(\beta)} R_{0}+O\left(R^{-3 / 2}\right) .
\end{gathered}
$$

Proof. The first four asymptotic equalities (16) are established just as in threedimensional elasticity [1, Ch. III, §2.3] by means of (14) and (15). To prove $(16)_{5}$, we replace $(13)_{2}$ and $\Delta u_{3}^{(\beta)}=-k_{\beta}^{2} u_{3}^{(\beta)}$ (from $\left.(12)_{2}\right)$ in (9), which becomes

$$
\left(k^{2}-k_{1}^{2}\right) u_{3}^{(1)}+\left(k^{2}-k_{2}^{2}\right) u_{3}^{(2)}+\operatorname{div} w^{(1)}=0 .
$$

We now apply grad to both sides above and use the equality grad div $=$ curl curl $+\Delta$, $(6)_{1},(13)_{1}$, and the substitutions $\Delta U^{(\beta)}=-k_{\beta}^{2} U^{(\beta)}$ (from $\left.(11)_{1}\right)$ to find that

$$
\left(k^{2}-k_{1}^{2}\right) \operatorname{grad} u_{3}^{(1)}+\left(k^{2}-k_{2}^{2}\right) \operatorname{grad} u_{3}^{(2)}-k_{1}^{2} U^{(1)}-k_{2}^{2} U^{(2)}=0 .
$$

Taking the scalar product of $R_{0}$ with both sides above and then using $(15)_{2}$ yields

$$
i k_{1}\left(k^{2}-k_{1}^{2}\right) u_{3}^{(1)}+i k_{2}\left(k^{2}-k_{2}^{2}\right) u_{3}^{(2)}=k_{1}^{2} R_{0} \cdot U^{(1)}+k_{2}^{2} R_{0} \cdot U^{(2)}+O\left(R^{-3 / 2}\right) .
$$

On the other hand, in view of $(13)_{1},(16)_{2}$ and (12), from (9) it follows that

$$
\left(k^{2}-k_{1}^{2}\right) u_{3}^{(1)}+\left(k^{2}-k_{2}^{2}\right) u_{3}^{(2)}+i k_{1} R_{0} \cdot U^{(1)}+i k_{2} R_{0} \cdot U^{(2)}=O\left(R^{-3 / 2}\right) .
$$

We adjoin this equality to (17) multiplied by $i$ to form a system of simultaneous linear equations for $R_{0} \cdot U^{(\beta)}$ :

$$
\begin{aligned}
& i k_{1} R_{0} \cdot U^{(1)}+i k_{2} R_{0} \cdot U^{(2)}=-\left(k^{2}-k_{1}^{2}\right) u_{3}^{(1)}-\left(k^{2}-k_{2}^{2}\right) u_{3}^{(2)}+O\left(R^{-3 / 2}\right), \\
& i k_{1}^{2} R_{0} \cdot U^{(1)}+i k_{2}^{2} R_{0} \cdot U^{(2)}=-k_{1}\left(k^{2}-k_{1}^{2}\right) u_{3}^{(1)}-k_{2}\left(k^{2}-k_{2}^{2}\right) u_{3}^{(2)}+O\left(R^{-3 / 2}\right) .
\end{aligned}
$$

Since, by $(3), k_{1} \neq k_{2}$ and $k_{1} k_{2} \neq 0$, this system has the unique solution

$$
R_{0} \cdot U^{(\beta)}=i k_{\beta}^{-1}\left(k^{2}-k_{\beta}^{2}\right) u_{3}^{(\beta)}+O\left(R^{-3 / 2}\right) .
$$


Now, by $(16)_{1},(18)$, and the properties of the triple vector product,

$$
\begin{aligned}
R_{0} \times\left(R_{0} \times U^{(\beta)}\right) & =O\left(R^{-3 / 2}\right)=\left(R_{0} \cdot U^{(\beta)}\right) R_{0}-U^{(\beta)} \\
& =i k_{\beta}^{-1}\left(k^{2}-k_{\beta}^{2}\right) u_{3}^{(\beta)} R_{0}-U^{(\beta)}+O\left(R^{-3 / 2}\right),
\end{aligned}
$$

from which we obtain $(16)_{5}$.

For $x \in \partial K_{R}$, the components of the boundary moment and shear stress vector $T u$ are (see [3], with $\nu$ regarded as the vector $\left(\nu_{1}, \nu_{2}, 0\right)^{\mathrm{T}}$ )

$$
\begin{gathered}
(T u)_{\alpha}=h^{2}\left[\lambda(\operatorname{div} w) R_{0}+2 \mu \frac{\partial w}{\partial R}+\mu R_{0} \times \operatorname{curl} w\right]_{\alpha}, \\
(T u)_{3}=\mu R_{0} \cdot w+\frac{\partial u_{3}}{\partial R} .
\end{gathered}
$$

Theorem 3. If $x \in \partial K_{R}$ and $u$ is a regular solution of (2) in $S^{-}$satisfying the radiation conditions (14) and (15), then for $R$ large Tu satisfies the estimates

$$
\begin{gathered}
{\left[T U^{(\beta)}-i h^{2}(\lambda+2 \mu) k_{\beta} U^{(\beta)}\right]_{\alpha}=O\left(R^{-3 / 2}\right),} \\
{\left[T U^{(3)}-i h^{2} \mu k_{3} U^{(3)}\right]_{\alpha}=O\left(R^{-3 / 2}\right),} \\
U^{(\beta)} \cdot U^{(3)}=O\left(R^{-2}\right) .
\end{gathered}
$$

Proof. In view of $(19)_{1},(11)_{2},(16)_{2}$ and $(14)_{2}$,

$$
\begin{aligned}
\left(T U^{(\beta)}\right)_{\alpha} & =h^{2}\left[\lambda\left(\operatorname{div} U^{(\beta)}\right) R_{0}+2 \mu \frac{\partial U^{(\beta)}}{\partial R}\right]_{\alpha} \\
& =i h^{2} k_{\beta}\left[\lambda\left(R_{0} \cdot U^{(\beta)}\right) R_{0}+2 \mu U^{(\beta)}\right]_{\alpha}+O\left(R^{-3 / 2}\right) .
\end{aligned}
$$

Using the triple product $R_{0} \times\left(R_{0} \times U^{(\beta)}\right)$ and taking $(16)_{1}$ into account, we establish the first estimate (20). The second estimate is derived similarly, by means of $(19)_{1}$, $(11)_{3},(14)_{2}$, and $(16)_{2,3}$. Finally, from the equality

$$
U^{(3)} \times\left(R_{0} \times U^{(\beta)}\right)=\left(U^{(3)} \cdot U^{(\beta)}\right) R_{0}-\left(R_{0} \cdot U^{(3)}\right) U^{(\beta)},
$$

$(14)_{1}$ and $(16)_{1,3}$ we deduce $(20)_{3}$.

\section{UNIQUENESS OF THE SOLUTION}

The preceding results can now be combined to answer the question of uniqueness for the solutions of the exterior Dirichlet and Neumann problems.

Theorem 4. If conditions (3) and the radiation conditions (14) and (15) hold, then each of $\left(\mathrm{D}^{-}\right),\left(\mathrm{N}^{-}\right)$has at most one regular solution.

Proof. The assertion is proved if we show that for $u=0$ or $T u=0$ on $\partial S$ the system (2) has only the trivial solution $u=0$ in $S^{-}$.

The procedure used to establish the reciprocity relation for bending of plates with transverse shear deformation $[2, \S 2.1]$, applied to a regular solution $u$ of the homogeneous problem $\left(\mathrm{D}^{-}\right)$or $\left(\mathrm{N}^{-}\right)$in the bounded domain $S^{-} \cap K_{R}$, leads to the equality [3]

$$
\int_{\partial K_{R}}(\bar{u} \cdot T u-u \cdot T \bar{u}) d s=0 .
$$


First we evaluate all the terms occurring in the expression for $\bar{u} \cdot T u$ on $\partial K_{R}$ arising from (10) and (19) for $R$ large. Thus, by (14) $)_{1}$ and $(20)_{1}$,

$$
\bar{U}^{(\beta)} \cdot T U^{(\beta)}=i h^{2}(\lambda+2 \mu) k_{\beta}\left|U^{(\beta)}\right|^{2}+O\left(R^{-2}\right) .
$$

Similarly,

$\bar{U}^{(1)} \cdot T U^{(2)}+\bar{U}^{(2)} \cdot T U^{(1)}=i h^{2}(\lambda+2 \mu)\left(k_{2} \bar{U}^{(1)} \cdot U^{(2)}+k_{1} \bar{U}^{(2)} \cdot U^{(1)}\right)+O\left(R^{-2}\right)$.

By $(14)_{1}$ and $(20)$,

$$
\bar{U}^{(\beta)} \cdot T U^{(3)}=O\left(R^{-2}\right), \quad \bar{U}^{(3)} \cdot T U^{(\beta)}=O\left(R^{-2}\right) .
$$

By $(14)_{1}$ and $(20)_{2}$,

$$
\bar{U}^{(3)} \cdot T U^{(3)}=i h^{2} \mu k_{3}\left|U^{(3)}\right|^{2}+O\left(R^{-2}\right) .
$$

By $(19)_{2},(15)_{1}$ and $(16)_{3}$,

$$
\bar{v}^{(\beta)} \cdot T U^{(3)}=\mu \bar{u}_{3}^{(\beta)} R_{0} \cdot U^{(3)}=O\left(R^{-2}\right) .
$$

By $(19)_{2}$ and (15),

$$
\begin{aligned}
\left(\bar{v}^{(1)}\right. & \left.+\bar{v}^{(2)}\right) \cdot\left(T v^{(1)}+T v^{(2)}\right) \\
& =\mu\left(\bar{u}_{3}^{(1)}+\bar{u}_{3}^{(2)}\right)\left(\frac{\partial u_{3}^{(1)}}{\partial R}+\frac{\partial u_{3}^{(2)}}{\partial R}\right) \\
& =\mu\left(i k_{1}\left|u_{3}^{(1)}\right|^{2}+i k_{2}\left|u_{3}^{(2)}\right|^{2}+i k_{2} \bar{u}_{3}^{(1)} u_{3}^{(2)}+i k_{1} \bar{u}_{3}^{(2)} u_{3}^{(1)}\right)+O\left(R^{-2}\right) .
\end{aligned}
$$

By $(19)_{2}$ and $(13)_{1}$, the remaining terms are

$$
\left(\bar{v}^{(1)}+\bar{v}^{(2)}\right) \cdot\left(T U^{(1)}+T U^{(2)}\right)=\mu\left(\bar{u}_{3}^{(1)}+\bar{u}_{3}^{(2)}\right) R_{0} \cdot\left(U^{(1)}+U^{(2)}\right) .
$$

Combining (22)-(28) with their complex conjugates, we compute the expression of the integrand in (21):

$$
\begin{aligned}
\bar{u} \cdot T u & -u \cdot T \bar{u} \\
= & 2 i\left\{h^{2}(\lambda+2 \mu)\left(k_{1}\left|U^{(1)}\right|^{2}+k_{2}\left|U^{(2)}\right|^{2}\right)+h^{2} \mu k_{3}\left|U^{(3)}\right|^{2}\right. \\
& +\mu\left(k_{1}\left|u_{3}^{(1)}\right|^{2}+k_{2}\left|u_{3}^{(2)}\right|^{2}\right)+h^{2}(\lambda+2 \mu)\left(k_{1}+k_{2}\right) \operatorname{Re}\left(U^{(1)} \cdot \bar{U}^{(2)}\right) \\
& \left.+\mu\left(k_{1}+k_{2}\right) \operatorname{Re}\left(u_{3}^{(1)} \bar{u}_{3}^{(2)}\right)+\mu \operatorname{Im}\left[\left(\bar{u}_{3}^{(1)}+\bar{u}_{3}^{(2)}\right) R_{0} \cdot\left(U^{(1)}+U^{(2)}\right)\right]\right\}+O\left(R^{-2}\right) .
\end{aligned}
$$

We need to estimate the terms without a modulus in (29).

By $(16)_{5}$ and $(15)_{1}$,

$$
\operatorname{Re}\left(U^{(1)} \cdot \bar{U}^{(2)}\right)=\left(k_{1} k_{2}\right)^{-1}\left(k^{2}-k_{1}^{2}\right)\left(k^{2}-k_{2}^{2}\right) \operatorname{Re}\left(u_{3}^{(1)} \bar{u}_{3}^{(2)}\right)+O\left(R^{-2}\right) .
$$

From (18),

$$
\begin{aligned}
& R_{0} \cdot U^{(1)}=i k_{1}^{-1}\left(k^{2}-k_{1}^{2}\right) u_{3}^{(1)}+O\left(R^{-3 / 2}\right), \\
& R_{0} \cdot U^{(2)}=i k_{2}^{-1}\left(k^{2}-k_{2}^{2}\right) u_{3}^{(2)}+O\left(R^{-3 / 2}\right) .
\end{aligned}
$$


If from the combination $\left[(31)_{1} \bar{u}_{3}^{(2)}+(31)_{2} \bar{u}_{3}^{(1)}\right]+\left[(31)_{1} \bar{u}_{3}^{(1)}+(31)_{2} \bar{u}_{3}^{(2)}\right]$ we now subtract its complex conjugate expression, we arrive at

$$
\begin{aligned}
\operatorname{Im}\left[\left(\bar{u}_{3}^{(1)}+\right.\right. & \left.\left.\bar{u}_{3}^{(2)}\right) R_{0} \cdot\left(U^{(1)}+U^{(2)}\right)\right] \\
= & \left.k_{1}^{-1}\left(k^{2}-k_{1}^{2}\right)+k_{2}^{-1}\left(k^{2}-k_{2}^{2}\right)\right] \operatorname{Re}\left(u_{3}^{(1)} \bar{u}_{3}^{(2)}\right) \\
& \quad+k_{1}^{-1}\left(k^{2}-k_{1}^{2}\right)\left|u_{3}^{(1)}\right|^{2}+k_{2}^{-1}\left(k^{2}-k_{2}^{2}\right)\left|u_{3}^{(2)}\right|^{2}+O\left(R^{-2}\right) .
\end{aligned}
$$

Hence, by (32), (30) and (8),

$$
\begin{aligned}
& h^{2}(\lambda+2 \mu)\left(k_{1}+k_{2}\right) \operatorname{Re}\left(U^{(1)} \cdot \bar{U}^{(2)}\right) \\
& +\mu\left(k_{1}+k_{2}\right) \operatorname{Re}\left(u_{3}^{(1)} \bar{u}_{3}^{(2)}\right)+\mu \operatorname{Im}\left[\left(\bar{u}_{3}^{(1)}+\bar{u}_{3}^{(2)}\right) R_{0} \cdot\left(U^{(1)}+U^{(2)}\right)\right] \\
& \quad=\mu\left[k_{1}^{-1}\left(k^{2}-k_{1}^{2}\right)\left|u_{3}^{(1)}\right|^{2}+k_{2}^{-1}\left(k^{2}-k_{2}^{2}\right)\left|u_{3}^{(2)}\right|^{2}\right]+O\left(R^{-2}\right) .
\end{aligned}
$$

Consequently, from (29) we obtain

$$
\begin{aligned}
& \bar{u} \cdot T u-u \cdot T \bar{u} \\
&=2 i {\left[h^{2}(\lambda+2 \mu)\left(k_{1}\left|U^{(1)}\right|^{2}+k_{2}\left|U^{(2)}\right|^{2}\right)+h^{2} \mu k_{3}\left|U^{(3)}\right|^{2}\right.} \\
&\left.+\mu\left(k_{1} k_{2}\right)^{-1} k^{2}\left(k_{2}\left|u_{3}^{(1)}\right|^{2}+k_{1}\left|u_{3}^{(2)}\right|^{2}\right)\right]+O\left(R^{-2}\right) .
\end{aligned}
$$

Replacing this in (21) and taking the limit as $R \rightarrow \infty$, in view of conditions (3) we deduce that

$$
\lim _{R \rightarrow \infty} \int_{\partial K_{R}}\left|U^{(j)}\right|^{2} d s=\lim _{R \rightarrow \infty} \int_{\partial K_{R}}\left|u_{3}^{(\beta)}\right|^{2} d s=0 .
$$

To complete the proof, we recall that the $U^{(j)}$ and $u_{3}^{(\beta)}$ satisfy the Helmholtz equations (11) or (12) and the corresponding radiation conditions (14) or (15), so, by the classical argument based on $(33)[4, \S 229]$, we conclude that

$$
U^{(j)}=u_{3}^{(\beta)}=0 \text { in } S^{-} .
$$

This means that $u=0$ in $S^{-}$, as required.

Remarks. (i) According to (10), (11) 1 and (12), the displacement field appears to have a spectrum of 5 'basic' time-harmonic oscillations. By $(11)_{2},(14)_{1}$ and $(16)_{1}$, the $U^{(\alpha)}$ are potential vector-functions whose component tangent to the wave front decays faster than the normal one away from the origin. Consequently, these vectors seem to describe asymptotically longitudinal oscillations. By $(11)_{3},(14)_{1}$ and $(16)_{3}, U^{(3)}$ is a solenoidal vector whose component normal to the wave front decays faster than the tangential one at infinity, so $U^{(3)}$ may be regarded as describing asymptotically transverse oscillations. The $v^{(\beta)}$ could be said to represent 'purely flexural' oscillations, with no equivalent in three-dimensional elasticity. Postulating the Sommerfeld radiation conditions (14) and (15) is equivalent to assuming that there are no sources at infinity for any of these basic spectral components.

(ii) The constants $k_{i}$ occurring in the expression of $u$ coincide with the wave numbers of the small-amplitude monochromatic waves propagating in the plate [5]. Under conditions (3) there are three distinct such waves. 


\section{REFERENCES}

1. V.D. Kupradze et al., Three-dimensional problems of the mathematical theory of elasticity and thermoelasticity, North-Holland, Amsterdam, 1979. MR 80h:73002

2. C. Constanda, A mathematical analysis of bending of plates with transverse shear deformation, Pitman Res. Notes Math. Ser. 215, Longman, Harlow, 1990. MR 91m:73016

3. P. Schiavone and C. Constanda, Oscillation problems in thin plates with transverse shear deformation, SIAM J. Appl. Math. 53 (1993), 1253-1263. MR 94g:73029

4. V.I. Smirnov, A course of higher mathematics, vol. 4, Pergamon Press, Oxford, 1964. MR 90k:00002c

5. C. Constanda and P. Schiavone, Flexural waves in Mindlin-type plates, Z. Angew. Math. Mech. 74 (1994), 492-493. CMP 95:03

Department of Mathematics, University of Strathclyde, Glasgow, United Kingdom

E-mail address: c.constanda@strath.ac.uk 